\title{
Dynamic observation of dislocation evolution and interaction with twin boundaries in silicon crystal growth using in - situ synchrotron X-ray diffraction imaging
}

\author{
M.G. Tsoutsouva ${ }^{\mathrm{a}, *}$, G. Regula ${ }^{\mathrm{b}}$, B. Ryningen ${ }^{\mathrm{c}}$, P.E. Vullum ${ }^{\mathrm{a}, \mathrm{c}}$, N. Mangelinck-Noël ${ }^{\mathrm{b}}$, \\ G. Stokkan ${ }^{\mathrm{c}}$ \\ a Dept. of Physics, Norwegian University Science and Technology, Høgskoleringen 5, Realfagbygget D5-170, 7491 Trondheim, Norway \\ ${ }^{\mathrm{b}}$ Aix Marseille Univ, Université de Toulon, CNRS, IM2NP, Marseille, France \\ ' SINTEF Industry, Trondheim, Norway
}

\begin{abstract}
The grown-in dislocation dynamics and interaction mechanisms with growth twins are investigated in- situ during the directional solidification of silicon crystal. The melting, solidification and cooling down

process is performed in a dedicated installation at the European synchrotron radiation facility and is fol- lowed by X-ray Bragg diffraction imaging techniques (X-ray topography) at the mesoscale in real-time.

Existing dislocations in the seed are observed to propagate in the up-grown crystal via replicas. They ex- pand vertically with the moving solid-liquid interface being always aligned perpendicular to the growth

front. During the solidification process when they meet a growth twin lamella ( $\Sigma 3\{111\})$, they neither

pile-up nor transmit through the boundary. They are blocked by the twin, but they continue to move laterally behind the growth front due to the thermomechanical stresses in the system. The existence of dis- locations at the solidliquid interface, their evolution and interaction with twin boundaries is discussed,

as growth proceeds, based on a detailed crystallographic analysis of the system.

Keywords:

Structural defects, Growth from melt, Directional solidification, X-ray topography
\end{abstract}

\section{Introduction}

Solar cells based on directionally solidified multi-crystalline silicon (mc-Si) ingots offer an attractive conversion efficiency/low production cost ratio. However, the inevitable generation of structural defects during the growth process limits the PV performance. Among the different types of defects, it is well established that dislocation clusters have the most detrimental impact because they gather impurity atoms and act as recombination sites for minority carriers, as well as shunt regions for majority carriers [1,2]. To control, and ideally prevent the cluster formation, and thus improve the quality of the solidified ingots, it is essential to understand the dislocation behaviour during the growth and cooling down process steps.

Several works can be found in the literature dealing with the formation and evolution of dislocation clusters in directionally so-

\footnotetext{
* Corresponding author.

E-mail addresses:
}

maria.tsoutsouva@ntnu.no（M.G. Tsoutsouva), lidified silicon crystals, investigating the cooled down ingot [38]. Ryningen et al. [9] studied horizontal slices taken along the height of an ingot and suggested a mechanism where: i) dislocations are generated locally at sources e.g. grain boundaries (GBs), or triple junctions $[10,11]$ in the vicinity of the growing interface, ii) then, they are pulled to the interface by image forces following the growth front, iii) multiplication can occur due to single ended Frank-Read sources and iv) as growth proceeds clusters are formed. The dislocations within a cluster were found to arrange in preferable crystallographic directions while most of the etch pits were circular, indicating that dislocation lines were parallel to the growth direction. This (or a similar mechanism) is supported by experimental observations where dislocation clusters form volumes like cylinders in the growth direction. However, the dislocation final arrangement results from the growth and post-growth movement, multiplication, interaction, recovery and is likely very different from the original configuration at high temperature. In general, dislocations present in the crystal are exposed to a screening effect aiming to reduce their individual elastic energy. Their attraction, repulsion and rearrangement in more stable and lower energy configurations are thermodynamically favored. This phenomenon is amplified when external forces, such 
as thermo-mechanical stresses, exist [12]. Chikawa [13] studied in- situ, at the melting point $\left(\mathrm{T}_{\mathrm{m}}\right)$, dislocations interacting with the

growth front perpendicularly. He found that dislocations of Burgers vector $1 / 2<110>$ which is common, are unstable at the growth interface at $\mathrm{T}<\mathrm{T}_{\mathrm{m}}$. They are not able to follow the front, with increasing growth rate, during the solidification process unless they form immobile composite types. Composite dislocations having the same line vector but different Burgers vectors (but not opposite) interact and form sessile dislocations with Burgers vectors of type $<100>$. The Chikawa's work is seminal and one could think that it is relevant for the directional solidification process (DS) as well as for the Dash necking process of Czochralski silicon (CZ).

Modeling and simulation studies have also been performed to understand the dislocation dynamics during Si solidification [1417]. Very recently, Zhou et al. [18] carried out molecular dynamics simulations to study the dislocation formation mechanism at the crystal/melt interface. They showed that dislocations form stochastically at the interface and in the case of strain-free growth, the probability of their formation is the highest during growth along the $<111>$, second highest during growth along $<112>$, and much lower along $<110>$ and $<100>$ directions in $\mathrm{Si}$.

As growth proceeds dislocations also interact with GBs in mc$\mathrm{Si}$ and all these phenomena occur during growth; i) behind the growth front, driven by the thermomechanical stresses that arise from thermal gradients or edge effects and ii) during cooling down where residual stresses can be released by glide as well as climb [19]. Lantreibecq et al. [20] plastically deformed casted Si TEM samples in-situ at high temperature and they found that dislocations are highly mobile above $900^{\circ} \mathrm{C}$ at low stress levels (4MPa). Indeed, they can move more than $1 \mathrm{~mm}$ per hour making the interaction between them and with GBs unavoidable. Examining a sample taken from a mono-like Si ingot, they found dislocations having an edge character to align one on top of the other, along the growth direction, forming recombination active small angle grain boundaries (SAGBs). In contrast with SAGBs, twins were not seen to act as obstacles, absorb or interact with mobile dislocations. They explained that by making the assumption that the dislocations were moving in planes parallel to twin boundaries. Yonenaga et al. [21] generated dislocations by a scratch after bending a mc-Si sample at $800^{\circ} \mathrm{C}$. Using the etch-pit and the x-ray topography techniques ex-situ, they observed dislocations to be stopped and piledup at twin $(\Sigma 3\{111\})$ boundaries under $\sim 18 \mathrm{MPa}$ applied stress. Moreover, new dislocations were found to be generated at the twin boundary for applied stress beyond $15 \mathrm{MPa}$ and it was attributed to the increased local stress concentration due to the pile-ups. The dislocation-grain boundary (GB) interaction mechanisms have been studied a lot in metals and alloys in the past decades by static and dynamic investigations since they play an important role for the mechanical properties of the material $[22,23]$. However, these works investigate the impact of annealing or deformation twins, and not growth twins as in $\mathrm{mc}-\mathrm{Si}$, on the behavior of dislocations under plastic deformation of the crystal. So, there is no direct correlation with the interaction of dislocations with twin boundaries formed during solidification, at very high temperatures (melting point $\mathrm{T}_{\mathrm{m}}$ ) and low level of applied stress.

It is practically impossible to decompose the involved mechanisms leading to the final dislocation arrangement by the ex-situ investigation of the cooled down ingot. In the present work, the grown-in dislocation dynamics and their interaction with growth twins are studied in-situ and in real-time during the directional solidification (DS) of Si. A Si crystal is melted, solidified and cooled down while the whole process is followed by synchrotron X-ray Bragg diffraction imaging (X-ray topography). This is the most appropriate characterization technique for in-situ mesoscopic visualization and investigation of crystal structure deformation and in particular the one due to dislocations [24]. The defect generation, evolution and interaction, during the crystal growth, is discussed based on a detailed crystallographic analysis of the system, providing an insight into the grown-in dislocation behavior at the mesoscale. The outcome of this work could be also relevant to other crystalline materials grown by the DS method.

\section{Experimental procedure}

The sample discussed in the present manuscript was taken from an ingot composed of several crystals with specific coincident site lattice (CSL) relationships (i.e $\Sigma 3\{111\}, \Sigma 3\{211\}, \Sigma 27\{552\}$ and $\Sigma 27\{511\})$. The ingot was directionally solidified in a Bridgman pilot-scale furnace (Crystalox DS250) using appropriate seeding to generate the above-mentioned particular GBs, of interest to study the interaction with dislocations during the growth process. The seeds were precisely oriented along specific crystallographic orientations with the aid of a high accuracy Laue X-ray system. While melting the charge, approximately $50 \%$ of the seed thickness was melted away. During solidification, the up-grown crystal took the initial orientation of the remaining seed producing the artificially designed GBs. Further details about the ingot preparation will be presented in an upcoming paper. For the present work, a vertical slice, was cut from the ingot along the $\Sigma 3\{211\}$ and thinned down to $\sim 320 \mu \mathrm{m}$ thickness by grinding and polishing. From the bottom of this slab, a small rectangular piece including the nonmelted seed and the specific GB was laser cut. After a final etching in a CP5 solution $\left(\mathrm{HNO}_{3}: \mathrm{HF}: \mathrm{CH}_{3} \mathrm{COOH} 5: 1: 2.5\right)$ to finalize the thickness and to remove the damage from the grinding/polishing /laser cutting steps, the sample dimensions were $40 \mathrm{~mm} \times$ $8 \mathrm{~mm} \times 0.3 \mathrm{~mm}(\mathrm{~h} \times \mathrm{w} \times \mathrm{t})$. This Si piece was then housed inside two pyrolytic BN plates, one containing a cavity corresponding to the sample shape and thickness, that were held together with Mo clamps (Fig. 1a). The sample was transferred to the European synchrotron radiation facility (ESRF, ID19 beamline) in Grenoble, France for the in-situ experiment. It was partially melted (keeping part of the initial seeds), directionally solidified (Fig. 1b, c) and cooled down in the high-temperature furnace of the dedicated IM2NP installation named GaTSBI (growth at high temperature observed by X-ray synchrotron beam imaging). The high temperature furnace was equipped with two resistive heating elements (top and bottom) and the experiment was performed under dynamic vacuum $\left(\sim 10^{-6}\right.$ mbar $)$. The sample was first heated by applying the same temperature up to $1373 \mathrm{~K}$ at the bottom and top heating resistances of the furnace. Beyond $1373 \mathrm{~K}$, a temperature gradient of $30 \mathrm{~K} / \mathrm{cm}$ was imposed between the two heating resistances until partial directional melting from top to bottom. After the stabilization of the solid/liquid interface in the lower part of the field of view, a cooling rate of $1 \mathrm{~K} / \mathrm{min}$ was applied on both heaters to start the directional solidification, from bottom to top. During the whole process, a synchrotron X-ray polychromatic beam passed through the Si sample and a Bragg diffraction spot (X-ray topograph) was continuously recorded.

Diffraction images contain information about the level of crystal perfection/deformation and the lattice defect formation such as dislocations and GBs [25,26]. Further information regarding the installation and previously obtained results can be found elsewhere [27-30]. Prior to the present experiments, the imaging system was upgraded to implement an indirect detector (scintillator coupled with a CMOS camera), replacing the traditional X-ray sensitive films, to record the Bragg diffraction images (Fig. 1d) [31]. The diffraction spot image covers a $8.8 \mathrm{~mm}^{2}$ field of view and is recorded on a $2048 \times 2048$ PCO edge camera which gives a pixel size of $4.3 \mu \mathrm{m}$. Only one diffraction spot image (one diffraction vector $\vec{g}$ ) can be recorded in real-time during the process. A spatial resolution of $4.3 \mu \mathrm{m}$ was used and allowed characterizing the strain field created by single dislocations and their dynamics. 


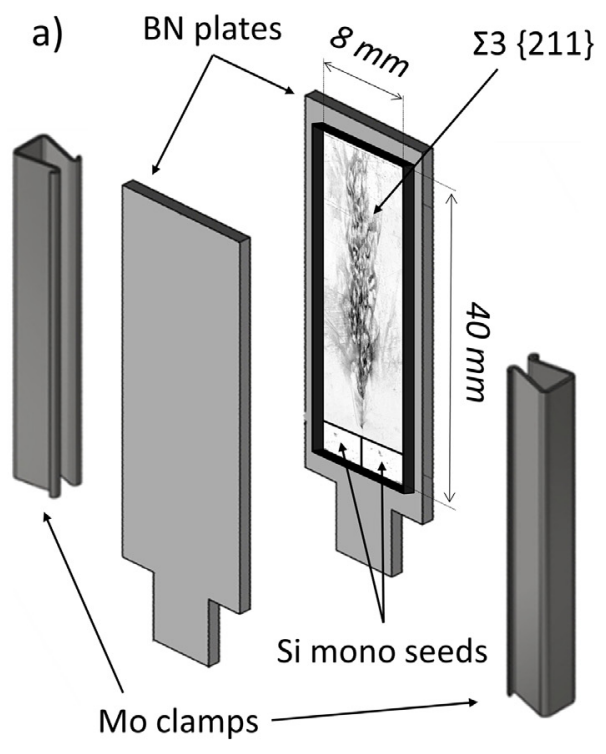

b)

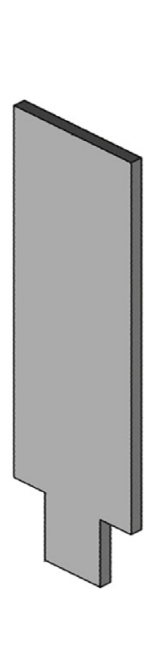

c)

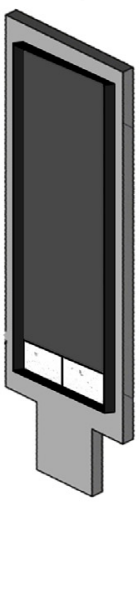

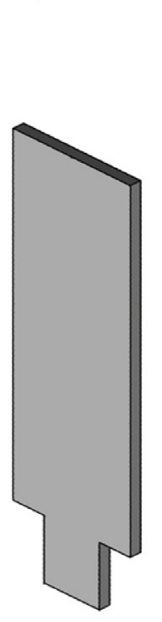

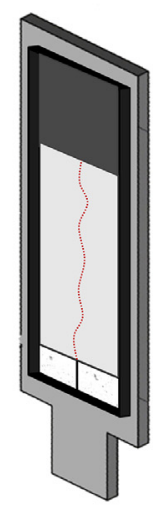



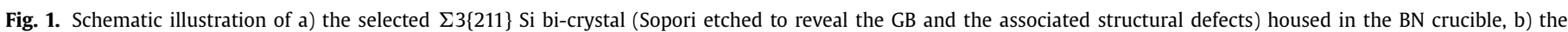

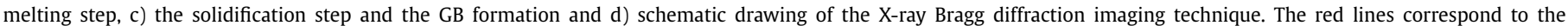
diffracted beams while $\overrightarrow{\boldsymbol{g}}$ is the diffraction vector. The vertical 022 Laue spot is continuously recorded on a PCO Edge 4.2 CCD camera.

Films can also be used to record several Laue spots simultaneously (Fig. 1d). The important benefit of using the camera instead of the $\mathrm{X}$-ray film is the much higher time resolution $(\sim 3 \mathrm{~s}$ for the camera, several min for the film) that allows following in details the defect dynamics and dislocation motion. Only dislocations that are not extinct, due to their relative Burgers vectors with the selected diffraction vector, can be observed. Indeed, a Laue spot corresponds to a diffraction vector $\vec{g}$ and thus to a specific crystallographic plane family of the sample. If the Burgers vector is perpendicular to the diffraction vector, the dislocation is extinct or present a weak contrast in the spot according to the invisibility criterion, $\vec{g} \cdot \vec{b}=0[32]$. After solidification, the sample was analyzed ex-situ by electron backscatter diffraction (EBSD) on a FEG-SEM JEOL JSM 7001F equipped with a HKL Nordlys camera using a $7 \mu \mathrm{m}$ step size. Inverse pole figure (IPF) maps were generated with respect to the three main directions relatively to the sample: perpendicular to the sample surface, transverse and along the growth direction. The CSL map reveals the orientation relationship between adjacent grains and boundaries with a special character as defined by the Brandon criterion [33]. Additionally, a plane view (parallel to the sample surface) transmission electron microscopy (TEM) sample was prepared using a dual-beam Helios G4 UX focused ion beam (FIB) from FEI. Carbon protection layers were deposited on the regions of interest prior to cutting out the TEM lamella. The lamella was cut out and transferred to a dedicated $\mathrm{Cu}$ half grid by a standard lift-out procedure. Coarse $\mathrm{Ga}^{+}$ion-beam thinning was performed at $30 \mathrm{kV}$ acceleration voltage. The final polishing of each side of the lamella was performed at 5 and $2 \mathrm{kV}$ to remove the amorphous Si at their surface due to Si redeposition and ion milling damage. TEM images and selected area diffraction pat- 
a)



e)

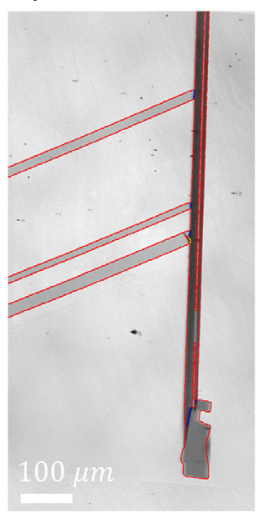

b)

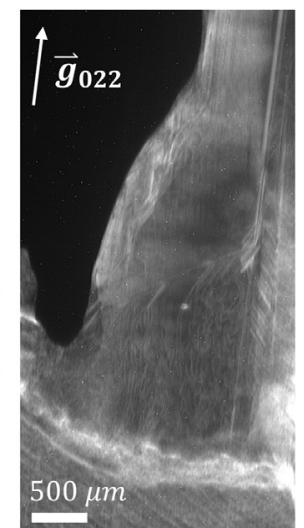

c)



d)

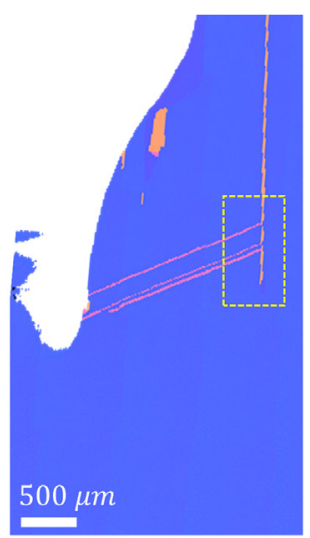

g)
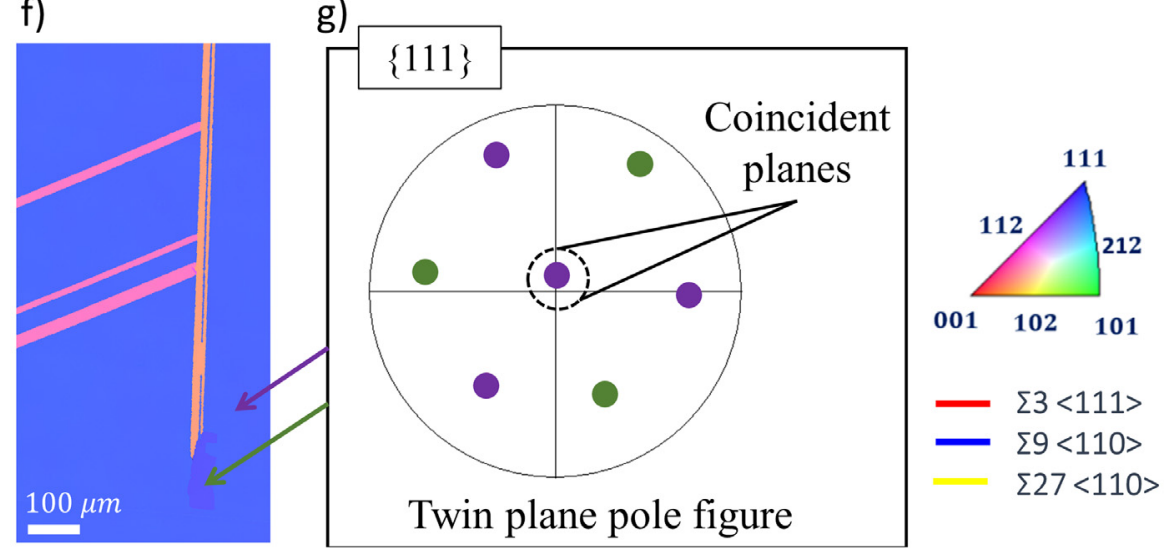

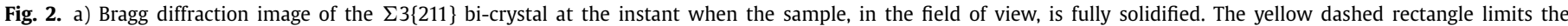

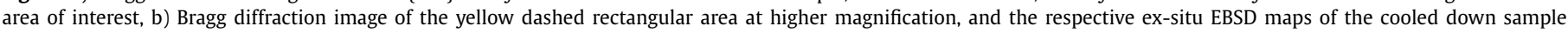

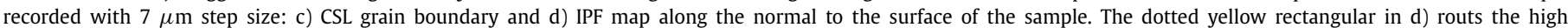

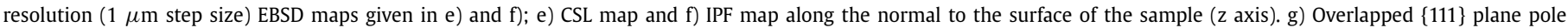
figures (viewed along the $z$ direction) of the small grain (green dots) and the matrix (purple dots) indicating the coincident $\{111\}$ plane.

terns were taken with a double Cs aberration corrected cold-FEG JEOL ARM 200F operated at $200 \mathrm{kV}$.

\section{Experimental results}

The Bragg diffraction image of the $\Sigma 3\{211\}$ bi-crystal, after melting and re-growth in the in-situ experiments, is shown in Fig. 2a. The vertical 022 reflection is selected to be followed during the whole process for the following reasons: i) $\{011\}$ is a common crystallographic plane family for both grains composing the bi-crystal. ii) As illustrated in the 022 spot in Fig. 1d, the diffraction images of the two adjacent grains are separated due to the slight misorientation (twist rotation around $\langle 211\rangle<1^{\circ}$ ) between them, whereas in $\overline{2} 20$ and $\overline{2} 42$ diffraction spots, the two images overlap hiding the details of the structural defects. iii) The intensity of the hkl reflection and the clear visibility of defects depend on the plane structure factor. Based on previous experimental observations, the 022 reflection gives better contrast and visibility of the structural defects than the $\overline{4} 2 \overline{2}$. iv) Dislocations having a Burgers vector and line vector parallel to the [011] growth direction are visible in the 022 and extinct in the $\overline{4} 2 \overline{2}$ reflection. These correspond to pure screw dislocations that are likely to form during the directional solidification process.

Even though a $\Sigma 3\{211\}$ bi-crystal has been grown, for the purpose of the present work, the analysis focuses only at the left edge of the sample (yellow dashed rectangular in Fig. 2a) sufficiently far from the GB to be able to consider that the interaction phenomena with the GB can be neglected during growth. The behavior of the specific GB during solidification is under study and will be discussed in another work. The Bragg diffraction image of the area of interest, recorded, and the respective ex-situ EBSD maps of the cooled down sample are shown in Fig. 2b, c and d. The up-grown crystal undertakes the initial orientation of the seed while double twinning events occur at the left edge of the sample. These lead to the formation of three diagonal twin lamellas by the alternation of the crystallographic orientations of the up-grown crystal. In addition to these diagonal twins a thin vertical twinned lamella is also visible. The area where the diagonal twins meet the vertical one was specifically analyzed with higher resolution EBSD, using a step size of $1 \mu \mathrm{m}$ instead of $7 \mu \mathrm{m}$ and the respective maps are presented in Fig. 2e, and f. The vertical twin starts from a small grain in the middle of the sample, which is in a twin relation with the matrix having a $\{111\}$ coincident plane almost parallel to the surface of the sample (Fig. $2 \mathrm{~g}$ ). As it can be seen in the pole figure map (Fig. $2 \mathrm{~g}$ ) there is no other $\{111\}$ common plane between them, denoting that all the GBs, formed between the small grain and the matrix, that are identified as $\Sigma 3$ in the CSL map, are incoherent (all GBs but symmetric $\Sigma 3\{111\}$ are defined as incoherent). The examination of this spontaneous nucleation event is out of the purpose of this work and does not affect neither the dynamics of the grown-in dislocations nor their interaction with the diagonal twins, so it will not be further discussed in the following. 
a)



$\mathrm{t}_{0}+121 \mathrm{~s}$

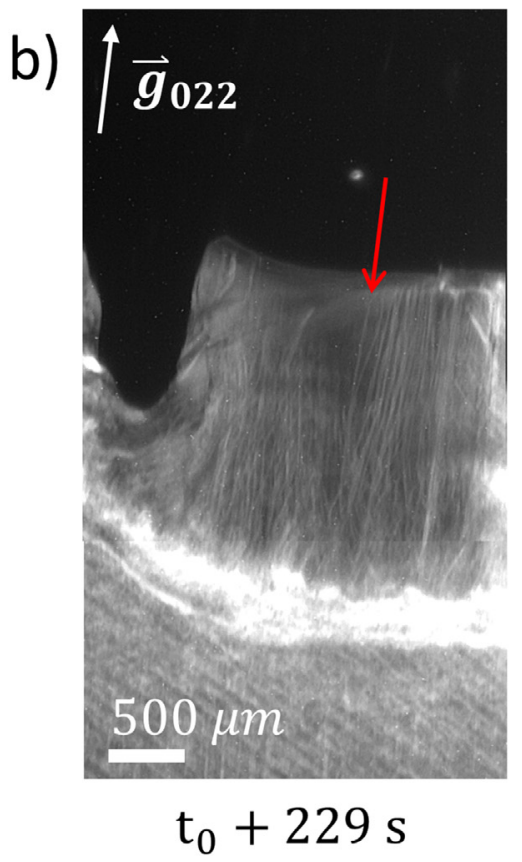

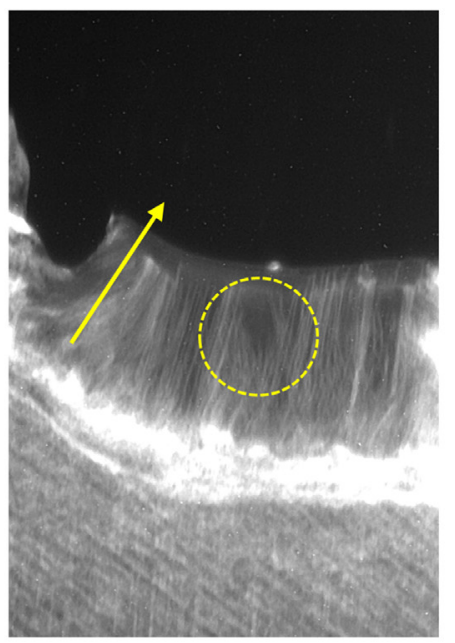

$\mathrm{t}_{0}+151 \mathrm{~s}$

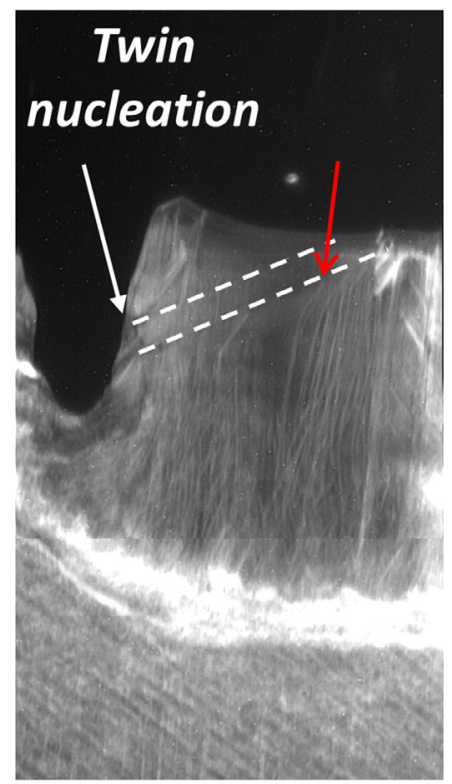

$\mathrm{t}_{0}+255 \mathrm{~s}$

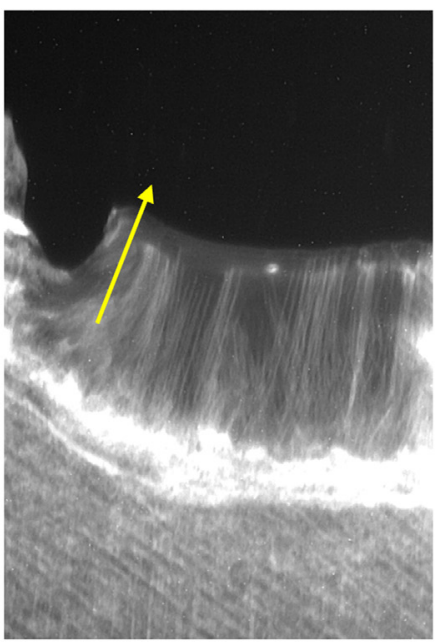

$\mathrm{t}_{0}+196 \mathrm{~s}$

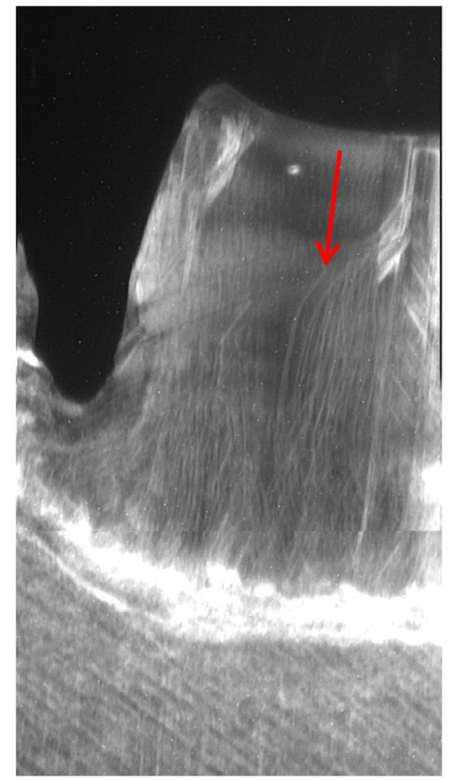

$\mathrm{t}_{0}+322 \mathrm{~s}$

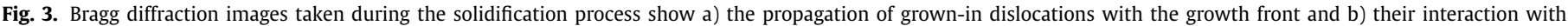

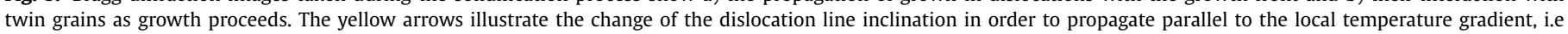

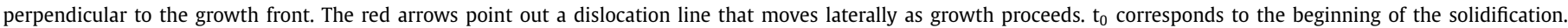
These images correspond to the area marked with a yellow dashed rectangular in Fig. 2a.

The focus of the present paper is on the dislocation transfer from the seed in the up-grown crystals, their evolution with the solid-liquid interface, as well as their interaction with twins. Corresponding experimental observations are described in the following. The Bragg diffraction images recorded at different times during the solidification process (Fig. 3) show the strain field due to single dislocation lines, ending at the solid-liquid interface and expanding along the growth direction (parallel to the local temperature gradient).

Close to the edge, where the solid-liquid interface is concave, dislocations are inclined relatively to the macroscopic vertical growth direction and propagate perpendicular to the growth front, parallel to the thermal gradient. As growth proceeds, the solidliquid interface becomes flatter and the dislocations bend, to be always perpendicular to the front (illustrated with yellow arrows in Fig. 3a). Away from the edge of the sample, closer to the cen- ter, where the solid/liquid interface is planar from the beginning of the solidification process, the dislocations are also aligned close to the growth direction although it is observed that they slightly deviate from this direction. Some of the dislocations remain connected to the solidification front and proceed with it throughout the image sequence, while others do not follow (marked with a yellow dashed circle in Fig. 3a). In addition to the joint propagation with the solid-liquid interface, dislocations are also observed to move laterally. This movement probably arises from the fact that the material is submitted to thermomechanical multiaxial stresses due to the applied axial temperature gradient, that also leads to the formation of a radial temperature gradient, as confirmed by the concave shape of the solid-liquid interface. Also, the sample is housed in between two BN plates that are held together with Mo clamps. When heating, the whole system expands ( $\mathrm{Si}, \mathrm{BN}$ and Mo have different thermal expansion coefficients) and thus a mechan- 


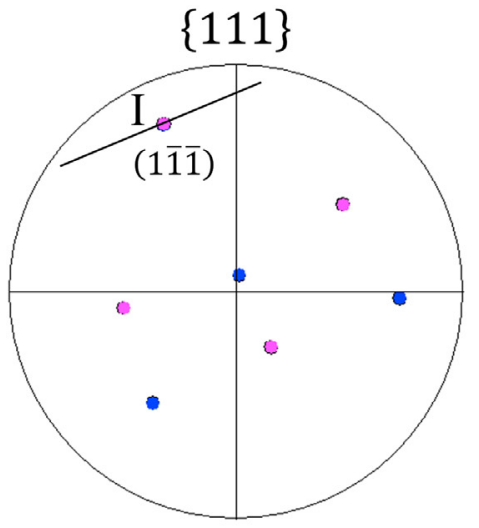

a)



b)



c)

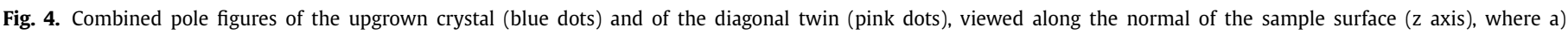


corresponds to the IPF orientation map illustrated in Fig. $2 \mathrm{f}$.

ical force applies on the front and back surface of the sample. To see this lateral movement, the reader could focus on the dislocation line indicated with a red arrow in Fig. $3 \mathrm{~b}$ and also watch the attached video in the supplementary material.

As the crystal continues to solidify, multiple twinning events occur at the edge forming thin twin lamellae (Figs. 2 and 3). This phenomenon has been observed and extensively discussed in our previous work [30] and is attributed to the sufficiently large undercooling in this area, that favors the formation of a twin nucleus. The GB formed between the upgrown crystal and the diagonal twin has been identified as $\Sigma 3<111>$, in the CSL map extracted from the EBSD measurements (red line in Fig. 2e). The atomic arrangement of the $\Sigma 3$ twin boundary can be either constructed by a $60^{\circ}$ or $180^{\circ}$ twist rotation about $<111>$ or $<211>$ axes, or a $70.5^{\circ}$ symmetric tilt from $<110>$ [34]. Consequently, the $\{111\},\{110\}$ and $\{211\}$ belong to the coincidence site lattice of $\Sigma 3$. The corresponding combined pole figures to the upgrown crystal (blue dots) and to the diagonal twin (pink dots) are shown in Fig. 4. In the same figure, the traces of the common planes are geometrically constructed and marked with Roman numerals. Plane traces are, by definition, perpendicular to the line that can be drawn between the point at the center of the pole figure, and the projection point of the respective shared pole $[35,36]$.

Fig. 4a presents the common $<111>$ rotation axis, which is equivalent to the existence of one common $\{111\}$ plane between the adjacent grains (pole I). The inclination of the respective plane trace is identical to the inclination of the investigated twin boundary (Figs. 2 and 3 ). The $\{110\}$ pole figure (Fig. $4 \mathrm{~b}$ ) reveals the presence of three common directions (poles II, III and IV) lying

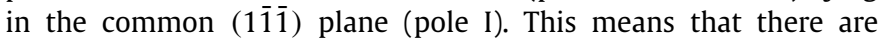
three common $\{111\}<110>$ slip systems available in the neighboring grains. Three common $\{211\}$ poles (Fig. $4 c$ ) exist in the investigated system, but none of the respective plane traces is parallel to the actual twin boundary. Consequently, the GB formed between the upgrown crystal and the diagonal twin, that nucleates at the edge of the sample, is a coherent and symmetric $\Sigma 3<111>$ with

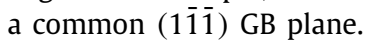

The formation of these diagonal twin lamellae affects the evolution of grown-in dislocations [30]. In the diffraction images presented in Fig. 3b and at higher magnification in Fig. 5, some grown-in dislocations do not appear above the twin i.e. they do not cross the boundary, while the contrast in the image does not change denoting the absence of pile-ups in this area. However, they continue to move laterally and bend, behind the growth front, adopting irregular arrangements.

\section{Discussion}

\subsection{Origin of dislocations}

The probable origin of the observed growth dislocations is the dislocations already present in the non-melted seed, thus neither representing the grain boundary source discussed by Ryningen et al. [9] nor the stochastic formation of dislocations at the s/l interface modelled by Zhou $[15,17,18]$. In the seed crystal two \{111\} glide planes are activated during the heating and the melting process; the diagonal (111) marked with a purple line in Fig. $6 \mathrm{a}$ and a purple pole in Fig. $6 \mathrm{~b}$ and the almost vertical ( $\overline{1} 1 \overline{1})$ marked with an orange line in Fig. 6a and an orange pole in Fig. 6c. The possible Burgers' vectors for a dislocation gliding in the (111) are: $\vec{b}=a / 2[\overline{1} 10], a / 2[01 \overline{1}], a / 2[10 \overline{1}]$ and the line vector

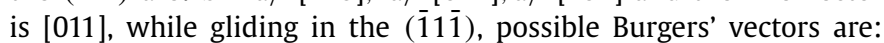
$\vec{b}=a / 2[011], a / 2[110], a / 2[10 \overline{1}]$, and line vector [011] where "a" is the lattice parameter of $\mathrm{Si}$ in the whole manuscript (Table 1a).

The slip system activation in the seed, during heating, has been previously investigated and it was shown that the sample preparation process induces dislocation sources via deformation and/or microcleavage at the edges of the seed [37]. In isothermal conditions, at approximately $1100^{\circ} \mathrm{C}$, dislocations nucleate, form halfloops and propagate throughout the entire width of the seed. The externally applied stress from the crucible sample-holder system activates specific crystallographic glide planes depending on the crystallographic orientation of the crystal. In this present case, the almost vertical (111) glide plane has the highest probability to be activated since it contains the direction of the applied thermal gradient. The (111) and (1 $\overline{1} \overline{1})$ should have similar probability, though the (111) should have the highest since it is closer to the vertical direction. However, only two slip systems, $((\overline{1} 1 \overline{1})$ and (111) glide planes), are activated during heating, as shown in Fig. 6, and likely are responsible for the dislocations visible in the up-grown crystal as the result of growth. In more general cases, the interface between the up-grown crystal and the seed can also act as a dislocation source via plastic deformation and or by the condensation of self-interstitials and vacancies as well as other dislocation types that may exist in the seed due to the initial sample preparation process, such as cellular dislocation arrangements.

A dislocation lying in the diagonal (111) with line vector along [110], found in the seed crystal, is selected for two-beam bright field (BF) TEM imaging for determination of its Burgers' vector. By tilting the specimen away from the [111] zone axis but keeping only one specific $\vec{g}$ vector in Bragg conditions, BF TEM images are 


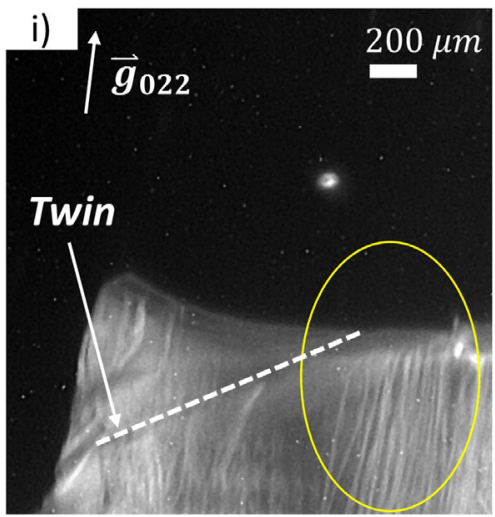

$\mathrm{t}_{0}+228 \mathrm{~s}$

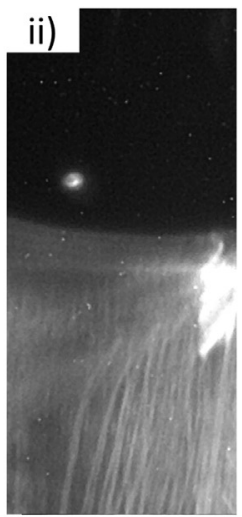

$t_{0}+264 s$

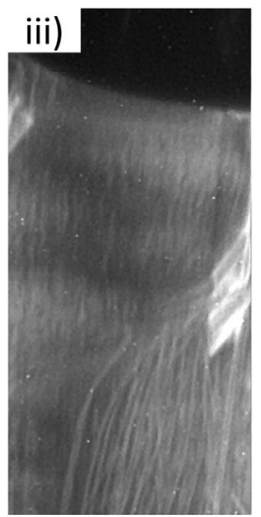

$t_{0}+336 s$

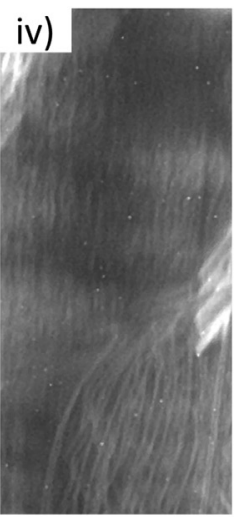

$t_{0}+426 s$



$t_{0}+515 s$


sponds to the beginning of solidification.

a)

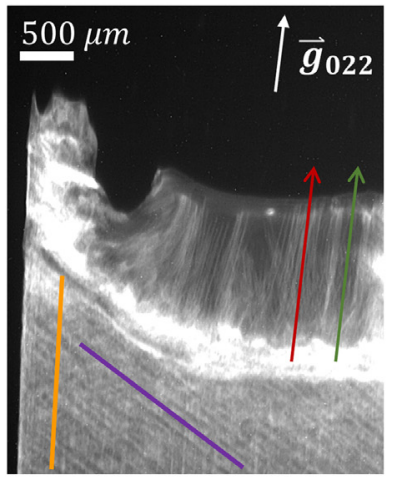

b)



c)

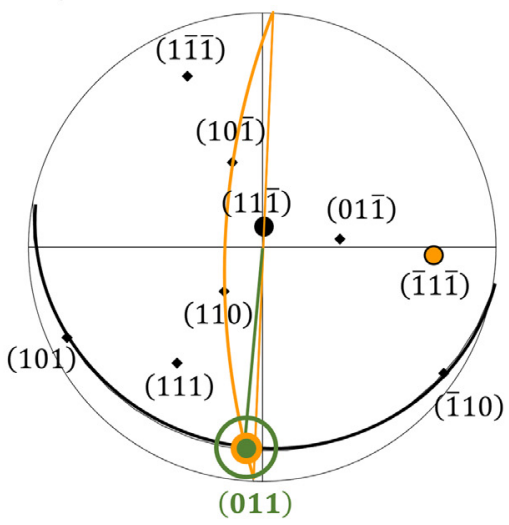

- Diagonal dislocation lines observed in the seed (glide plane (111)). Almost vertical dislocation lines observed in the seed (glide plane ( $\overline{1} 1 \overline{1})$ ).

$\longrightarrow 60^{\circ}$ grown-in dislocations with $\vec{b}=\mathrm{a} / 2[\overline{1} 10]$ and line vector along [011]

$\longrightarrow$ screw grown-in dislocations with $\vec{b}=a / 2$ [011] and line vector [011]



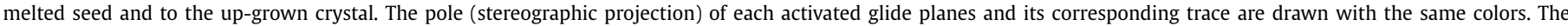

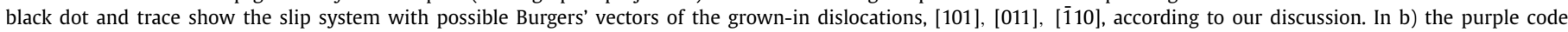

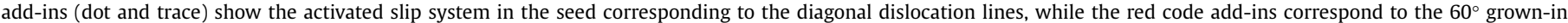

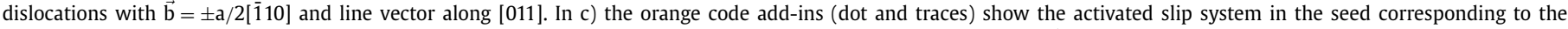
almost vertical dislocation lines, while the green code add-ins correspond to the screw grown-in dislocations with $\vec{b}= \pm a / 2[011]$ and line vector along [011].

recorded (Fig. 7b). In two-beam condition, the criterion $\vec{g} \cdot \vec{b}=0$ [32] is fulfilled for $\vec{g}=[224]$ denoting that the sole possible Burgers vector, in the corresponding glide plane (111) is $\vec{b}= \pm \mathrm{a} / 2[\overline{1} 10]$ and thus the dislocation has a pure screw character. The dislocation segment shown in the TEM images is short because the [110] is not perfectly parallel to the surface plane (11) $)$ and thus it has been cut during the preparation of the lamella. Unfortunately, no dislocation segment lying in the ( $\overline{1} 1 \overline{1})$ was found in the thin TEM lamella, therefore its Burgers vector could not be identified.

\subsection{Grown-in dislocations}

In the following it is examined how a dislocation, that belongs to one of the two slip systems in the seed (purple and orange in Fig. $6 \mathrm{~b}$ and c), can be transferred in the up-grown crystal and propagate with the front. To achieve that, the possible grown-in dislocation glide planes and characters are analyzed. As seen above and based on the crystallographic orientation of the up-grown crystal (Fig. 6b and c), which is the same as the seed, and observing the line vector parallel to the [011] direction, the grown-in dislocations can either glide in the vertical $(\overline{1} 1 \overline{1})$ or the $(11 \overline{1})$ glide plane which is almost parallel to the surface of the sample (perpendicular to our field of view). Some, clear dislocation lines move laterally below the growth front in the Bragg diffraction images (Fig. 3 and video in the supplementary material), due to thermomechanical stresses in the system. Knowing that the sample thickness is about $300 \mu \mathrm{m}$ and the (111) plane inclination to the sample surface is $70.5^{\circ}$ ( $19.5^{\circ}$ perpendicular to the sample surface), the maximum lateral distance that a dislocation could cover, gliding in this system within the sample thickness is $\tan 19.5^{\circ} \times 300 \mu \mathrm{m}=$ $106.2 \mu \mathrm{m}$. Since a dislocation cannot terminate in the middle of a crystal, it can either be pinned at structural defects such as precipitates, which is not observed, or go out of the thin sample reaching its front or back side while gliding in the (i11) plane. Besides, the grown-in dislocations, highlighted by the red arrow in Fig. 3b, are observed to move laterally covering distances bigger than $350 \mu \mathrm{m}$ being always present in the field of view. This directly implies that they glide in the (11) $)$ plane which is almost parallel to the surface 
Table 1

Summary of the observed activated slip systems and their respective dislocation characters in a) the seed crystal and b) the up-grown crystal. The color code add-ins are equivalent to Fig. 6a, b and c.

\begin{tabular}{|c|c|c|}
\hline \multicolumn{3}{|c|}{ a) Dislocations in the seed } \\
\hline Glide plane & Line vector & Burgers vector \\
\hline$(\mathbf{1 1 1})$ & {$[\overline{\mathbf{1} 10]}$} & $\mathrm{a} / 2[\overline{\mathbf{1}} \mathbf{0}]$ \\
\hline$(111)$ & {$[\overline{1} 10]$} & $\mathrm{a} / 2[01 \overline{1}]$ \\
\hline$(111)$ & {$[\overline{1} 10]$} & $\mathrm{a} / 2[10 \overline{1}]$ \\
\hline$(\overline{\mathbf{1}} \overline{\mathbf{1}})$ & {$[\mathbf{0 1 1}]$} & $\boldsymbol{a} / \mathbf{2}[\mathbf{0 1 1}]$ \\
\hline$(\overline{1} 1 \overline{1})$ & {$[011]$} & $a / 2[110]$ \\
\hline$(\overline{1} 1 \overline{1})$ & {$[011]$} & $a / 2[10 \overline{1}]$ \\
\hline
\end{tabular}

\begin{tabular}{|c|c|c|c|}
\hline \multicolumn{3}{|c|}{ b) Grown-in dislocations } \\
\hline Glide plane & Line vector & Burgers vector & Type \\
\hline$(11 \overline{1})$ & {$[011]$} & $\mathrm{a} / 2[\overline{1} 10]$ & $60^{\circ}$ \\
\hline$(11 \overline{1})$ & {$[011]$} & $\mathrm{a} / 2[011]$ & screw \\
\hline$(11 \overline{1})$ & {$[011]$} & $\mathrm{a} / 2[101]$ & $60^{\circ}$ \\
\hline
\end{tabular}

of the sample. A dislocation that glides in the (11) $\overline{1}$ ) plane having a line vector along [011] can have one of the following possible Burgers vector directions, regardless of the signs: [110], [011], [101] (Table $1 \mathrm{~b}$ and also illustrated with a black dot and trace in Fig. 6b, c). Thus, this dislocation can either be characterized as $60^{\circ}$ with $\vec{b}=a / 2[\overrightarrow{1} 10]$ line vector [011] or $\vec{b}=a / 2[101]$ line vector [011] (red color dots and line in Fig. $6 \mathrm{~b}$ ) or as screw with $\vec{b}=\mathrm{a} / 2$ [011] line vector [011] (green color dot and line in Fig. 6c).

Assuming that the dislocations already present in the seed expand in the up-grown crystal during the solidification process two scenarios may exist: i) the dislocation initially glides in the diagonal (111) plane (purple add-ins in Fig. 6a and b) and ii) the dislocation initially glides in the almost vertical ( $\overline{1} 1 \overline{1})$ plane (orange add-ins in Fig. 6a and c).

- A dislocation that glides in the (111) plane can have 3 different Burgers vector directions: [110], [011] , [101] . When it expands or simply replicate in the up-grown crystal it has to change its glide plane from (111) to (11) $)$ and this is only possible for dislocations having a Burgers vector that belongs to both of these planes. The only possibility is $\vec{b}=\mathrm{a} / 2[\overline{1} 10]$ (red circled pole in Fig. 6b) and thus, the character of the grown-in dislocation is $60^{\circ}$ with $\vec{b}=\mathrm{a} / 2[\overrightarrow{1} 10]$ and line vector along [011] (Table 1). This is in accordance with the Burgers vector identification by the two-beam bright field (BF) TEM imaging.

- Similarly, to the previous reasoning, a dislocation that glides in the $(\overline{1} 1 \overline{1})$ can have 3 possible Burgers vectors:
[011], [110], [101] ]. Expanding in the up-grown crystal it must move to the (11 $\overline{1})$ glide plane and only a dislocation having a Burgers vector $\vec{b}=\mathrm{a} / 2[011]$ (green circled pole in Fig. $6 \mathrm{c}$ ) which direction is common in the $(\overline{1} 1 \overline{1})$ and $(11 \overline{1})$ glide planes can cross slip. Thus, the character of the grown-in dislocation is pure screw with $\vec{b}=a / 2[011]$ and line vector [011] (Table 1).

It has been shown that two dislocation characters, that are initially in the seed, $\vec{b}=\mathrm{a} / 2[\overline{1} 10]$ and $\vec{b}=a / 2[011]$ can replicate in the up-grown crystal and expand during the solidification process, following the moving solid/liquid interface. In general, grownin dislocations must intersect the interface to minimize its energy while the direction of their line depends on the shape of the growth front, the orientation of the grown crystal and their Burgers vector. In the present case, they either align close to the growth direction or slightly deviate towards the lowest atomic potential (Peierls valley) which contribute to the minimization of their energy. This means that dislocations should have a big screw component, or be even pure screw as shown earlier, otherwise the angle would not be $90^{\circ}$. The dislocation equilibrium line position is the one which limits its line tension, thus minimizes its length and maximizes its screw segment. The observed grown-in dislocation alignment perpendicular to the growth front can be explained by two theories $[12,19,38]$ :

1. The zero-force theorem: when a dislocation meets a free crystal surface or a boundary, additionally to the forces to which it is exposed, it experiences an image force along its dislocation 
(a)

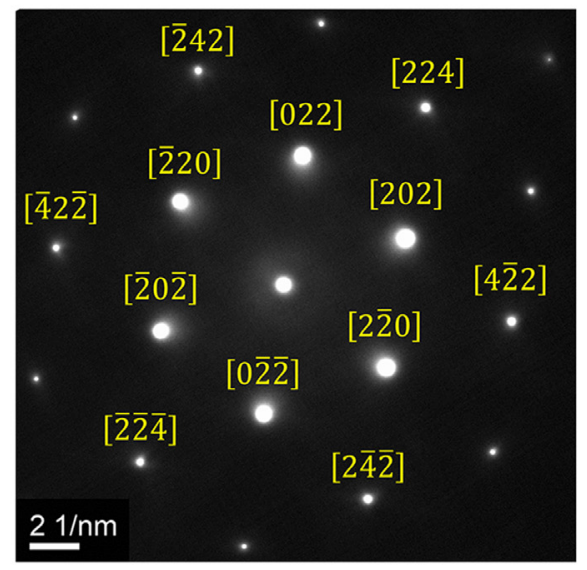

(b)
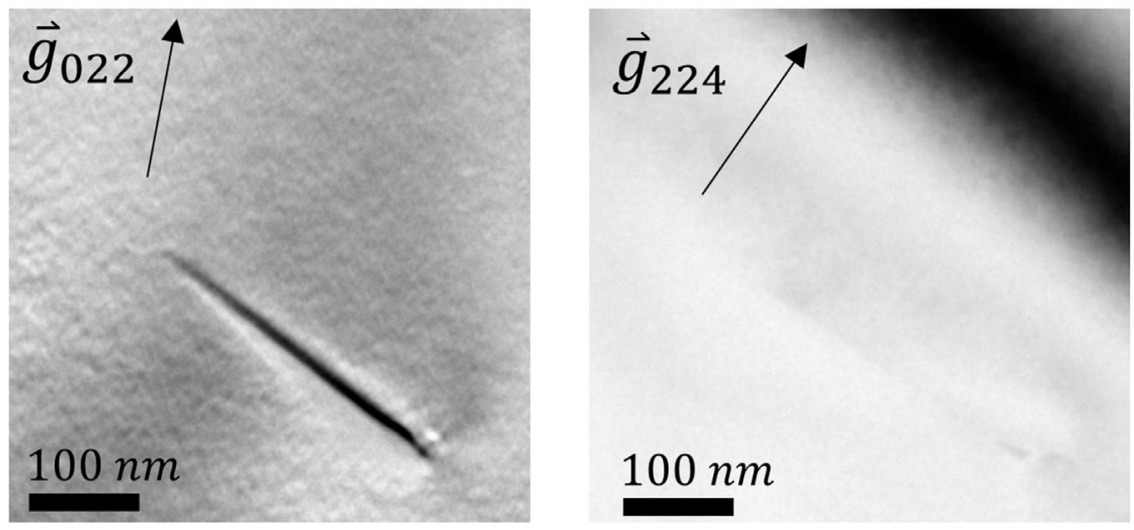

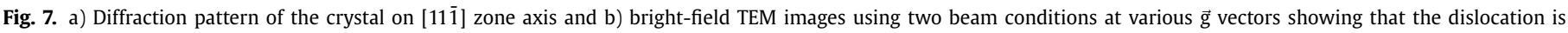
extinct for $\vec{g}=[224]$.

line. Because of the less rigid structure at the free surface, the dislocation is attracted towards the free surface.

2. The minimum-energy theorem: a dislocation adopts a preferred dislocation line, in order to minimize its energy within a growth layer, such as the solid-liquid interface in the present case.

In reality, based on Lothe's theorem [39], both approaches lead to the same favorable direction that a dislocation follows when ending at the solid-liquid interface.

The findings of this work are different from Chikawa's [13] observations who suggests that only composite type of dislocations can be present at the solid-liquid interface and propagate as the crystal grows. In the present experiment the fact that dislocations

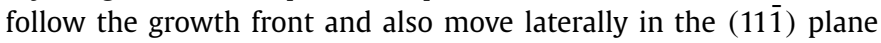
parallel to the sample surface, is a clear indication that they cannot be sessile, meaning that they are regular dislocations and not composite. One explanation for this discrepancy could be the different experimental conditions. In the present case many of the observed dislocations have the same Burgers vector direction since they come from the same source, which is only the one edge of the sample (dislocations in the activated diagonal slip system in the seed). Consequently, they cannot recombine forming the characteristic "hairpin-shape" as in the experiment performed by Chikawa, they cannot get out of the front or back side of the sample, they cannot terminate inside the crystal and thus they have to follow the growth front, even if they are mobile. This may be a representative situation for directional solidification growth (different from Dash necking) where a grain boundary emits dislocations moving in the same direction under a stress field. These dislocations have the same Burgers vector direction and indeed are able to propagate with the growth front.

\subsection{Grown-in dislocation interaction with twin boundaries}

As growth proceeds, the grown-in dislocations meet the diagonal twin that nucleates at the edge of the sample and expands towards the inner part of the sample (Fig. 5). There are four possible dislocation-GB interaction models: 1) direct dislocation transmission through the GB in the adjacent grain via cross slip, 2) direct transmission via dissociation of the incoming dislocation, where a residual dislocation remains in the $G B, 3$ ) indirect transmission via absorption of the incident dislocation, reaction with the GB and/or incorporation, subsequent dislocation nucleation and re-emission in the adjacent grain, 4) no transmission, where in that case the GB act as a sink and the dislocation is incorporated. A GB can also solely act as sources for the nucleation and emission of dislocations but this is not discussed since it is not relevant to the present observations [40-42]. The parameters that regulate the reaction are the character of the incident dislocation, the coincidence 
lattice sites between both grains and the interfacial plane of the $\mathrm{GB}$ as well as the applied and local stress fields.

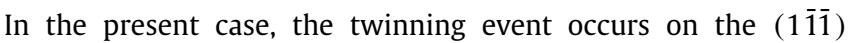
common GB plane between the parent crystal and the twin (Fig. 4a). As discussed above and illustrated in Fig. 6b, c, the

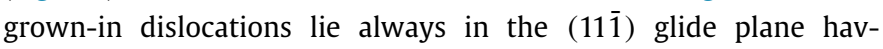
ing a Burgers' vector either $\vec{b}= \pm \mathrm{a} / 2[10]$ (60 dislocation) or $\vec{b}$ $=$

\section{1}

$\pm a / 2[011]$ (screw dislocation). A comparison between the crystallographic orientation of the adjacent grains shows that there is no incoming and outgoing slip system sharing either of these directions as a common line of intersection at the level of the GB.

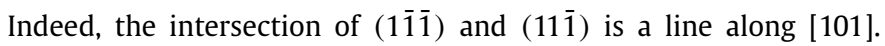
Therefore, the grown-in dislocations cannot be directly transmitted in the adjacent grain either through the GB via cross slip or via dissociation of the incoming dislocation. The strain field of the incoming dislocations is not visible above the twin in the Bragg diffraction image (Fig. 5), meaning that an indirect transmission reaction also does not occur. Furthermore, the contrast in the image at the meeting point does not change denoting the absence of pile-ups in this area. Thus, the dislocations interact with the GB, they are locked at the twin interface, as the growth front proceeds away from the dislocation line. At last, they bend, lying always in

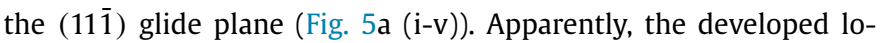
cal stress at the intersection is not enough to newly generate a dislocation from the twin GB into the adjacent grain [21]. The dislocation bending results from the lateral movement of the locked dislocations due to thermal stresses in the system arising from the solidification process that forces dislocations to continue gliding, adopting irregular arrangements. Interestingly they continue to move inside the cooling down sample close to the melting temperature while the front advances higher up in the sample.

Likely, when the dislocation impinges in the boundary, it is shredded by atomic redistribution and then absorbed by the twin which acts as a sink. This may lead to the distortion at the core of the dislocation, as well as the formation of disconnections namely interfacial steps at the twin boundary [43]. Sangid et al. [42] performed molecular dynamics simulations to study the dislocation twin boundary interaction mechanisms. In one of their investigated cases, they describe the incorporation of an incident $60^{\circ}$ dislocation type in a twin boundary leading to a complete blockage, due to the formation of a stable sessile Frank partial dislocation. At the location of impingement, the structural atomic arrangement is lost, and the twin becomes less coherent. This mechanism could be one possible explanation of the dislocation locking at the twin boundary observed in the present experiment. However, the resolution of the Bragg diffraction images is far not enough to allow distinguishing the dissociation of a dislocation and the interaction with the twin at the atomic scale.

Anyways, it becomes clear that even though coherent $\Sigma 3$ twin boundaries do not induce any distortion in the crystal and do not present any recombination activity in $\mathrm{mc}-\mathrm{Si}$, they can interact with dislocations during the solidification process. In particular, in the present work, it is demonstrated that the transmission of dislocations does not take place in all cases. A twin boundary may block their propagation by a dislocation absorption mechanism that very likely induces interfacial atomic steps at the boundary. Given that the interfacial atomic structure has a direct impact on the electrical behavior of CSL GBs in silicon [44], this could also explain why certain twins, even of high crystallographic quality as $\Sigma 3$, are seen in the literature to be recombination active.

\section{Conclusions}

The grown-in dislocation evolution and interaction with twin boundaries have been investigated under dynamic conditions during the directional solidification, by Synchrotron X-ray Bragg diffraction imaging (white beam X-ray topography). Dislocations, pre-existing in the seed, due to the sample preparation procedure as well as to the slip system activation during heating, are replicated in the up-grown crystal via a cross slip mechanism. As a second step, grown-in dislocations expand along the growth direction, intersecting with the solid-liquid interface to minimize their total length (free energy), being parallel to the local temperature gradient. Two possible dislocation characters have been identified, either $60^{\circ}$ with $\vec{b}=\mathrm{a} / 2[\overline{1} 10]$, line vector [011] or screw with $\vec{b}=a / 2[011]$, line vector [011] that both glide in the (11) plane. Thus, it is demonstrated that in seeded directional solidification growth, glissile dislocations can follow the front, which may also be a representative situation for dislocations emitted by grain boundaries.

When meeting a growth $\Sigma 3\{111\}$ twin boundary plane, some dislocations are not observed either to directly or indirectly transmit or pile-up at the intersection. The crystallographic analysis showed that there is no common Burgers' vector at two glide planes on either sides of the twin boundary. Interestingly, it looks like the dislocations are absorbed by the boundary, they are locked at the interface and as growth advances further, they continue to glide adopting irregular arrangements. This could probably explain why certain coherent $\Sigma 3$ twins are seen in the literature to be electrically active.

\section{Declaration of Competing Interest}

The authors declare that they have no known competing financial interests or personal relationships that could have appeared to influence the work reported in this paper.

\section{Acknowledgments}

The work is supported by the Research Council of Norway's ENERGIX program in the frame of the INSIDES project (255326) and by the ANR within the CrySaLID Project ( $\mathrm{N}^{\circ}$ ANR-14-CE05-004601 ). The Research Council of Norway is further acknowledged for the support to NTNU NanoLab through the Norwegian Micro- and Nano-Fabrication Facility, NorFab (197411/V30). The TEM work was carried out on NORTEM infrastructure, Grant 197405, TEM Gemini Centre, Norwegian University of Science and Technology (NTNU), Norway. The ESRF (European Synchrotron Radiation Facility) ID19 team is greatly acknowledged for support during the X-ray imaging experiments. We would also like to thank Pål Tetlie and Emil Grove Dyrvik for their contribution to the sample preparation, Guillaume Reinhart, Maike Becker and Hadjer Ouaddah for their valuable assistance during the elaboration of the solidification experiments, Fabrice Guittonneau and Laurent Barrallier for performing the EBSD measurements and Marisa Di Sabatino for fruitful discussions.

\section{Supplementary materials}

Supplementary material associated with this article can be found, in the online version, at doi:10.1016/j.actamat.2021.116819.

\section{References}

[1] S. Woo, M. Bertoni, K. Choi, S. Nam, S. Castellanos, D.M. Powell, T. Buonassisi, H. Choi, An insight into dislocation density reduction in multicrystalline silicon, Sol. Energy Mater. Sol. Cells. 155 (2016) 88-100, doi:10.1016/j.solmat. 2016.03.040.

[2] T. Strauch, M. Demant, P. Krenckel, S. Riepe, S. Rein, Analysis of grain structure evolution based on optical measurements of mc-Si wafers, Sol. Energy Mater. Sol. Cells. 182 (2018) 105-112, doi:10.1016/j.solmat.2018.03.009.

[3] M.M. Kivambe, G. Stokkan, T. Ervik, B. Ryningen, O. Lohne, The microstructure of dislocation clusters in industrial directionally solidified multicrystalline silicon, J. Appl. Phys. 110 (2011) 063524, doi:10.1063/1.3641978. 
[4] G. Stokkan, Y. Hu, Ø. Mjøs, M. Juel, Study of evolution of dislocation clusters in high performance multicrystalline silicon, Sol. Energy Mater. Sol. Cells. 130 (2014) 679-685, doi:10.1016/j.solmat.2014.02.034.

[5] D. Kohler, A. Zuschlag, G. Hahn, On the origin and formation of large defect clusters in multicrystalline silicon solar cells, Sol. Energy Mater. Sol. Cells. 120 (2014) 275-281, doi:10.1016/j.solmat.2013.09.018.

[6] G. Stokkan, A. Song, B. Ryningen, Investigation of the grain boundary character and dislocation density of different types of high performance multicrystalline silicon, crystals. 8 (2018) 341. https://doi.org/10.3390/cryst8090341.

[7] M. Trempa, I. Kupka, C. Kranert, T. Lehmann, C. Reimann, J. Friedrich, Evolution of grain structure and recombination active dislocations in extraordinary tall conventional and high performance multi-crystalline silicon ingots, J. Cryst. Growth. 459 (2017) 67-75, doi:10.1016/j.jcrysgro.2016.11.030.

[8] D. Oriwol, M. Trempa, L. Sylla, H.S. Leipner, Investigation of dislocation cluster evolution during directional solidification of multicrystalline silicon, J. Cryst. Growth. 463 (2017) 1-9, doi: 10.1016/j.jcrysgro.2017.01.027.

[9] B. Ryningen, G. Stokkan, M. Kivambe, T. Ervik, O. Lohne, Growth of dislocation clusters during directional solidification of multicrystalline silicon ingots, Acta Mater. 59 (2011) 7703-7710, doi: 10.1016/j.actamat.2011.09.002.

[10] D. Oriwol, M. Hollatz, M. Reinecke, Control of dislocation cluster formation and development in silicon block casting, Energy Proc. 27 (2012) 66-69, doi:10. 1016/j.egypro.2012.07.030.

[11] N. Chen, S. Qiu, J. Huang, G. Du, G. Liu, Study of dislocations in the minicrystal- lized regions in multicrystalline silicon grown by the directional solidification method, Crystals 6 (2016) 130, doi: 10.3390/ cryst6100130.

[12] P. Rudolph, Dislocation patterning and bunching in crystals and epitaxial layers - a review, Cryst. Res. Technol. 52 (2017), doi:10.1002/crat.201600171.

[13] J. Chikawa, Live X-ray topography and crystal growth of silicon, Jpn. J. Appl. Phys. 38 (1999) 4619, doi:10.1143/JJAP.38.4619.

[14] M. Ishimaru, S. Munetoh, T. Motooka, K. Moriguchi, A. Shintani, Moleculardynamics studies on defect-formation processes during crystal growth of silicon from melt, Phys. Rev. B. 58 (1998) 12583-12586, doi:10.1103/PhysRevB.58. 12583.

[15] N. Zhou, X. Wu, X. Wei, L. Zhou, Y. Wan, D. Hu, A molecular dynamics study of nucleation of dislocation in growth of silicon from melt, J. Cryst. Growth. 443 (2016) 15-19, doi:10.1016/j.jcrysgro.2016.03.017.

[16] J. Godet, P. Hirel, S. Brochard, L. Pizzagalli, Dislocation nucleation from surface step in silicon: the glide set versus the shuffle set, Phys. Status Solidi A. 206 (2009) 1885-1891, doi: 10.10 02/pssa.20 0881460.

[17] N. Zhou, X. Sui, X. He, S. Huang, L. Zhou, Nucleation of self-growth dislocations on growth front during the solidification process of silicon, J. Appl. Phys. 125(2019) 155108, doi: 10.1063/1.5088125.

[18] N. Zhou, X. Wei, L. Zhou, Formation of dislocations in the growth of silicon along different crystallographic directions-a molecular dynamics study, crys-
tals. 8 (2018) 346. https://doi.org/10.3390/cryst8090346.

19] H. Klapper, Generation and propagation of defects during crystal growth, in: G. Dhanaraj, K. Byrappa, V. Prasad, M. Dudley (Eds.), Springer Handb. Cryst. Growth, Springer Berlin Heidelberg, Berlin, Heidelberg, 2010: pp. 93-132. https://doi.org/10.1007/978-3-540-74761-1_4.

[20] A. Lantreibecq, J.-Ph. Monchoux, E. Pihan, B. Marie, M. Legros, Subgrains, micro-twins and dislocations characterization in monolike Si using TEM and in-situ TEM, Mater. Today Proc. 5 (2018) 14732-14747, doi:10.1016/ j.matpr. 2018.03.063.

[21] I. Yonenaga, K. Kutsukake, Transmission behavior of dislocations against $\Sigma 3$ twin boundaries in Si, J. Appl. Phys. 127 (2020) 075107, doi:10.1063/1.5139972.

[22] N.V. Malyar, J.-S. Micha, G. Dehm, C. Kirchlechner, Dislocation-twin boundary interaction in small scale Cu bi-crystals loaded in different crystallographic di-
rections, Acta Mater 129 (2017) 91-97, doi:10.1016/j.actamat.2017.02.067.

[23] D. Wei, M. Zaiser, Z. Feng, G. Kang, H. Fan, X. Zhang, Effects of twin boundary orientation on plasticity of bicrystalline copper micropillars: a discrete dislocation dynamics simulation study, Acta Mater 176 (2019) 289-296, doi:10.1016/j. actamat.2019.07.007.

[24] A.N. Danilewsky, X-ray topography-more than nice pictures, Cryst. Res. Technol. 55 (2020) 2000012, doi:10.1002/crat.202000012.

[25] R.C. Burns, A.I. Chumakov, S.H. Connell, D. Dube, H.P. Godfried, J.O. Hansen, J. Härtwig, J. Hoszowska, F. Masiello, L. Mkhonza, M. Rebak, A. Rommevaux,

R. Setshedi, P.V. Vaerenbergh, HPHT growth and x-ray characterization of high- quality type IIa diamond, J. Phys. Condens. Matter. 21 (2009) 364224, doi:10. 1088/0953-8984/21/36/364224.

[26] A.R. Lang, The early days of high-resolution X-ray topography, J. Phys. Appl. Phys. 26 (1993) A1-A8, doi:10.1088/0022-3727/26/4A/001.

[27] M.G. Tsoutsouva, G. Stokkan, G. Regula, B. Ryningen, T. Riberi - Béridot G. Reinhart, N. Mangelinck-Noël, Random angle grain boundary formation and evolution dynamics during Si directional solidification, Acta Mater. 171 (2019) 253-260, doi:10.1016/j.actamat.2019.04.010.

[28] T. Riberi-Béridot, N. Mangelinck-Noël, A. Tandjaoui, G. Reinhart, B. Billia, T. Lafford, J. Baruchel, L. Barrallier, On the impact of twinning on the formation of the grain structure of multi-crystalline silicon for photovoltaic applications during directional solidification, J. Cryst. Growth. 418 (2015) 38-44, doi:10.1016/j.jcrysgro.2015.02.024.

[29] T. Riberi - Béridot, M.G. Tsoutsouva, G. Regula, G. Reinhart, F. Guittonneau, L. Barrallier, N. Mangelinck-Noël, Strain building and correlation with grain nucleation during silicon growth, Acta Mater. 177 (2019) 141-150, doi:10.1016/j. actamat.2019.07.035.

[30] M.G. Tsoutsouva, T. Riberi - Béridot, G. Regula, G. Reinhart, J. Baruchel, F. Guittonneau, L. Barrallier, N. Mangelinck-Noël, In situ investigation of the structural defect generation and evolution during the directional solidification of 110 seeded growth Si, Acta Mater. 115 (2016) 210-223, doi:10.1016/j.actamat. 2016.06.004.

[31] M. Becker, G. Regula, G. Reinhart, E. Boller, J.-P. Valade, A. Rack, P. Tafforeau, N. Mangelinck-Noël, Simultaneous X-ray radiography and diffraction topography imaging applied to silicon for defect analysis during melting and crystallization, J. Appl. Crystallogr. 52 (2019) 1312-1320, doi:10.1107/ S1600576719013050.

[32] M. Moore, S.G. Nailer, W.K. Wierzchowski, Optical and X-ray topographic studies of dislocations, growth-sector boundaries, and stacking faults in synthetic diamonds, Crystals 6 (2016) 71, doi:10.3390/cryst6070071.

[33] D.G. Brandon, The structure of high-angle grain boundaries, Acta Metall. 14 (1966) 1479-1484, doi:10.1016/0001-6160(66)90168-4.

[34] J.W. Jhang, T. Jain, H.K. Lin, C.W. Lan, Possible twinning operations during directional solidification of multicrystalline silicon, Cryst. Growth Des. 18 (2018) 2518-2524, doi:10.1021/acs.cgd.8b00115.

[35] E. Schmid, S. Würzner, C. Funke, T. Behm, R. Helbig, O. Pätzold, H. Berek, M. Stelter, The correlation between spatial alignment of dislocations, grain orientation, and grain boundaries in multicrystalline silicon, Cryst. Res. Technol. 47 (2012) 229-236, doi:10.1002/crat.201100373.

[36] E.-R. Carl, A. Danilewsky, E. Meissner, T. Geiger, Large- and small-angle grain boundaries in multi-crystalline silicon and implications for the evolution of grain boundaries during crystal growth. J. Appl. Crystallogr. 47 (2014) 19581965, doi:10.1107/S1600576714023061.

[37] M.G. Tsoutsouva, T. Riberi-Béridot, G. Regula, G. Reinhart, J. Baruchel, N. Mangelinck-Noël, In situ imaging of dislocation expansion in FZ-Si seeds during temperature ramp heating process, Phys. Status Solidi A. 215 (2018) 1700758, doi:10.1002/pssa.201700758

[38] Derek Hull, D.J. Bacon, Introduction to Dislocations, 5th Edition, Elsevier, Amsterdam, 2011 (n.d.)

[39] J. Lothe, V.L. Indenbom, V.A. Chamrov, Elastic field and self-force of dislocations emerging at the free surfaces of an anisotropic halfspace, Phys. Status Solidi B. 111 (1982) 671-677, doi:10.1002/pssb.2221110231.

[40] L. Priester, Grain Boundaries: From Theory to Engineering, Springer, Netherlands, 2013, doi:10.1007/978-94-007-4969-6.

[41] P. Chen, F. Wang, B. Li, Dislocation absorption and transmutation at 1012 twin boundaries in deformation of magnesium. Acta Mater. 164 (2019) 440-453, doi:10.1016/j.actamat.2018.10.064.

[42] M.D. Sangid, T. Ezaz, H. Sehitoglu, Energetics of residual dislocations associated with slip-twin and slip-GBs interactions, Mater. Sci. Eng. A. 542 (2012) 21-30, doi:10.1016/j.msea.2012.02.023.

[43] A. Serra, D.J. Bacon, Interaction of a moving twin boundary with perfect dislocations and loops in a hcp metal, Philos. Mag. 90 (2010) 845-861, doi:10.1080 14786430903023901.

[44] M.G. Tsoutsouva, P.E. Vullum, K. Adamczyk, M. Di Sabatino, G. Stokkan, Interfacial atomic structure and electrical activity of nano-facetted CSL grain boundaries in high-performance multi-crystalline silicon, J. Appl. Phys. 127 (2020) 125109, doi:10.1063/1.5130996. 